\title{
ANSCOMBE, LA EXPRESIÓN DE AUTOCONCIENCIA Y LA REGLA DE AUTORREFERENCIA
}

\author{
Javier Vidal* \\ Universidad de Concepción, Chile \\ fravidal@udec.cl
}

\begin{abstract}
Resumen
"The First Person" es el artículo en el que G. E. M. Anscombe sostiene la tesis de que "yo" no es una palabra referencial. Discuto, principalmente, su argumento contra la concepción indéxica de "yo" a partir del caso del usuario de "A", quien refiere a él mismo como la persona que cae bajo la observación especial del usuario de "A". Por un lado, propongo que un uso de "A" podría tener una referencia garantizada en un sentido semántico: un uso referencial de "A" como una descripción definida garantiza la referencia única a uno mismo. Por otro lado, la concepción indéxica queda intacta: la referencia de "A" no es fijada por la regla de autorreferencia, y de este modo "yo" y "A" no son palabras referencialmente indistinguibles. También examino la idea de que, a diferencia de "A", "yo" tiene una referencia garantizada en un sentido epistémico, siendo inmune a un error de identificación.
\end{abstract}

Palabras Clave: autorreferencia, autoconciencia, sobreveniencia, reflexivo indirecto, descripción definida, uso referencial, inmunidad a un error de identificación.

\section{Abstract}

"The First Person" is the paper where G. E. M. Anscombe supports the thesis that "I" is not a referring word. Mainly I deal with her argument against the indexical view of "I" from the scenario of the " $A$ " user, who refers to himself as the person who is under the special observation of the " $A$ " user. On the one hand, I put forward that a use of "A" might have a guaranteed reference in a semantic sense: a referential use of " $A$ " as a definite description guarantees the one reference to oneself. On the other hand, the indexical view is left untouched: the reference of " $A$ " is not fixed by the self-reference rule, and thus "I" and " $A$ " are not indistinguishable referring words. Also I explore the idea that unlike " $A$ ", "I" has a guaranteed reference in an epistemic sense, being immune to error through misidentification.

KEY WORDS: Self-Reference, Self-Consciousness, Supervenience, Indirect Reflexive, Definite Description, Referential Use, Immunity to Error through Misidentification.

Doctor en Filosofía. Profesor Asistente. Universidad de Concepción, Departamento de Filosofía, Beltrán Mathieu 15A, Concepción, Chile.E-mail: fravidal@udec.c 


\section{Introducción}

R⿴囗十

En The First Person, G. E. M. Anscombe sostiene que el pronombre personal "yo" no es una palabra referencial'. Como acertadamente expone B. Garrett (cf. 1998, pp. 95-99), la tesis de Anscombe puede verse como un ataque contra, entre otras concepciones referencialistas, la concepción de sentido común sobre "yo", la concepción indéxica según la cual la referencia de una emisión particular de "yo" resulta fijada por la siguiente regla de autorreferencia: una instancia dada de "yo" refiere a quienquiera que la produjo, es decir, el usuario de "yo". Garrett responde al ataque de Anscombe, que contiene esencialmente un argumento basado en el caso del usuario de una palabra especial "A": una palabra imaginaria que todo hablante usaría para referirse a él mismo a partir de la observación de su cuerpo y de sus acciones; una palabra que, a pesar de ser autorreferencial, según Anscombe, carece de lo que es específicamente genuino del uso de "yo" como una expresión de autoconciencia.

Garrett acusa a Anscombe de pasar de la dimensión semántica a la dimensión epistémica en el argumento sobre el usuario de "A", lo que podría ser inadmisible: una diferencia de carácter referencial entre "A" y "yo" es una diferencia semántica que no tiene que depender de consideraciones epistémicas sobre la identificación (y reidentificación) del referente. Pero creo que la interpretación de Garrett no es totalmente correcta. De entrada, no es correcta en la medida en que, como establezco en el diagnóstico general de su error, Anscombe no sostiene directamente que la cuestión de la identificación del referente es una condición necesaria para la referencialidad de "yo". Pero, la interpretación de Garrett tampoco es correcta en la medida en que el argumento de Anscombe se atiene a otro tipo de conexión legítima entre lo semántico y lo epistémico. La idea de fondo de Anscombe es, a mi modo de ver, que, teniendo que haber una conexión necesaria entre la referencia y el uso de una palabra so pena

1 Una vía rápida para deshacerse de la tesis de que "yo" no es una palabra referencial consistiría en argumentar desde la sintaxis a la referencia. En este sentido, Anscombe declara absurda la tarea afirmando que "nadie piensa que 'Está lloviendo' ('It is raining') contiene una expresión referencial, 'it"' (Anscombe 1981, p. 30). A esto, B. Garrett responde que el carácter no-referencial de tales usos de "it" es confirmado de otros modos, por ejemplo, al no poder inferir "Something is raining" desde "It is raining"; en cambio, sí es posible inferir "Someone is in pain" desde "I am in pain" (cf. Garrett 1994, p. 112). Ahora bien, puede afirmarse que eso no es conclusivo al poder inferir "It is raining at a place" desde "It is raining". Y también puede afirmarse que así como el carácter referencial de "it" no puede ser obtenido de la satisfacción de una regla como: si en París es emitido "It is raining" lo aseverado será verdadero si, y solo si, está lloviendo en París (pero "it" no refiere a París!), tampoco el carácter referencial de "yo" puede ser obtenido de la satisfacción de la regla: si X emite "I am in pain" lo aseverado será verdadero si, y solo si, X siente dolor (X no tiene que ser el referente de "I"). 
de que la referencia no juegue ningún rol en el uso $^{2}$, debería suceder que la expresión de autoconciencia mediante un uso de "yo" sobrevenga en la fijación de la referencia de "yo" mediante la regla de autorreferencia, en cuyo caso una diferencia con respecto a la expresión de autoconciencia entraña una diferencia con respecto a la fijación de la referencia. La fuerza del argumento sobre el usuario de " $\mathrm{A}$ " sería esta: dado el principio de sobreveniencia, si los usos de una palabra como "A" no expresan autoconciencia, pero su referencia es fijada por la regla de autorreferencia, entonces los usos de "yo", que sí expresan autoconciencia, no pueden tener una referencia que sea fijada por la regla de autorreferencia ${ }^{3}$.

En 1 y 2a examino por qué una explicación del significado de "yo" en términos del reflexivo indirecto "él mismo" no es satisfactoria, y eso deja espacio libre para hacer una comparación semántica entre "yo" y "A" en términos de la regla de autorreferencia. En $2 b$ muestro, contra Garrett, que un uso de "A" tiene referencia garantizada en un sentido netamente semántico, en el sentido -que de hecho Anscombe no contempla- de que un uso referencial de "A" como una descripción definida garantiza que el usuario de "A" solo habla de él mismo. Además, argumento, contra Anscombe, que no se ha probado que "A" y "yo" son palabras referencialmente indistinguibles, dado que una referencia garantizada mediante un uso referencial de "A" como una descripción definida excluye que la referencia de " $A$ " sea fijada por la regla de autorreferencia, lo que es semánticamente determinante de la referencia de "yo" según la concepción indéxica. En 2c analizo brevemente la tesis de que un uso de "yo", a diferencia de un uso de "A", tenga referencia garantizada en un sentido netamente epistémico, siendo inmune a un error de identificación, y concluyo que eso no tiene consecuencias para la referencialidad de "yo"; y termino con un diagnóstico general del error de Anscombe sobre la relación entre las dimensiones semántica y epistémica de un uso de "yo".

2 La exigencia de tal conexión es lo que la va a llevar a decir: "Con los nombres, o las expresiones que denotan (en el sentido de Russell) hay dos cosas que captar: el tipo de uso, y aquello a lo que se aplican de una a otra ocasión. Con 'yo' hay solo el uso" (Anscombe 1981, p. 32).

3 Si una propiedad $\mathrm{M}$ (por ejemplo, una propiedad mental) sobreviene en una propiedad $\mathrm{F}$ (por ejemplo, una propiedad física) entonces: si x es F, entonces necesariamente x es M. De esto se sigue que dos individuos $\mathrm{x}$ e y no pueden diferir con respecto a la posesión de la propiedad M sin diferir con respecto a la posesión de la propiedad F. Estoy formulando la conexión necesaria entre uso y referencia que Anscombre introduce, en términos de un principio de sobreveniencia de la expresión de autoconciencia en la fijación de la referencia mediante una regla de autorreferencia: si la referencia de una palabra " $x$ " es fijada mediante la regla de autorreferencia, entonces necesariamente un uso de " $x$ " expresa autoconciencia. De esto se sigue que dos palabras "yo" $y$ " $A$ " no pueden diferir con respecto a la expresión de autoconciencia sin diferir con respecto al modo como su referencia es fijada. 


\section{La cuestión del reflexivo indirecto}

En su artículo, Anscombe emprende inicialmente una vía que la conduce con rapidez a desestimar la concepción indéxica y, no obstante, se ve obligada a construir el caso del usuario de "A". Mi tesis es que ello es debido a que la objeción inicial depende de un concepto vacuo de autoconciencia. Anscombe introduce la regla de autorreferencia así: supongamos que el significado de "yo" consiste en que es la palabra que cada persona usa para hablar de ella. El problema es que las personas pueden usar términos mediante los que hablan de ellas y, con todo, no haya involucrado algún tipo de autoconciencia en el uso de esos términos. Por tanto, hay un sentido de 'hablar de uno' que no es una explicación suficiente de la autoconciencia del hablante. Por ejemplo, si Edipo dice que el asesino de Layo debe ser llevado ante la justicia, Edipo está hablando de él pero, con todo, no hay expresión de autoconciencia porque, diríamos, Edipo no sabe que él, él mismo, es la persona de la que está hablando. El primer sentido de 'hablar de uno', que no es expresivo de autoconciencia, es un sentido tal que, a pesar de la ignorancia de Edipo, si Edipo dice que el asesino de Layo debe ser llevado ante la justicia, la oración "Edipo dice que él debe ser llevado ante la justicia" es verdadera. El pronombre "él" cuyo referente es Edipo es aquí el pronombre reflexivo ordinario o directo, cuyo funcionamiento está regido por la claúsula de que puede ser sustitutido por términos correferenciales salva veritate. Por el contrario, el segundo sentido de 'hablar de uno', donde hay expresión de autoconciencia, es un sentido tal que, dada la ignorancia de Edipo, si Edipo dice que el asesino de Layo debe ser llevado ante la justicia, la oración "Edipo dice que él mismo debe ser llevado ante la justicia" es falsa. El pronombre "él mismo" cuyo referente es Edipo es aquí el pronombre reflexivo indirecto, para el que no rige la regla de sustitución salva veritate.

Por tanto, la explicación de que el primer sentido de 'hablar de uno' no exprese la autoconciencia del hablante propia de un uso de "yo" es que no considera el conocimiento que tiene el hablante de estar hablando de él mismo, porque el hecho de usar ciertos términos que refieren a él (como "el asesino de Layo") no garantiza que el hablante tenga ese tipo de conocimiento. Pero este es un concepto al menos vacuo, es decir, redundante de autoconciencia. En efecto, queda sin explicar en qué consiste el conocimiento que tiene el hablante de estar hablando de él mismo. Ciertamente, pueden aducirse ulteriores hechos semánticos que muestran la diferencia entre el uso del reflexivo directo y el indirecto: por ejemplo, si la oración con el reflexivo indirecto "X dice que él mismo es F" es verdadera, podemos inferir que X realizó una emisión de "Yo soy F", en cambio, no es legítima esa inferencia desde una análoga oración con el reflexivo directo. Pero seguimos estando ante una descripción de la diferencia semántica, no ante una explicación de la diferencia semántica que se haga cargo de un concepto no vacuo de autoconciencia.

Una prueba de que tal concepto de autoconciencia no es explicativo lo tenemos al evaluar la solución de L. O’Brien (cf. 1994, pp. 277-281). Supongamos que el significado de "yo" viene dado por la regla de autorreferencia donde el sentido de "hablar de uno' es el del reflexivo directo. Parece que hasta aquí tenemos autorreferencia pero 
no autorreferencia autoconsciente, por así decirlo. Ahora bien, O’Brien nos recuerda el hecho de que un hablante que realiza una emisión cualquiera sabe al realizarla que él mismo es quien la realiza. La conclusión es que cualquier hablante que realiza una emisión con "yo" lleva a cabo una autorreferencia autoconsciente, una referencia a él bajo un modo de presentación de primera persona. Sin embargo, la solución es fallida: tanto si Edipo emite la oración "El asesino de Layo debe ser llevado a la justicia", como la oración "Yo debo ser llevado ante la justicia", Edipo logra referirse a él en el sentido del reflexivo directo ${ }^{4}$ y a la vez sabe al realizarla, bajo un modo de presentación de primera persona, que él mismo es quien realiza la emisión. Con todo, sigue habiendo una diferencia según la cual la emisión de "El asesino de Layo debe ser llevado ante la justicia" no cuenta como una expresión de autoconciencia y, en cambio, "Yo debo ser llevado ante la justicia" sí lo hace. Solo en el segundo caso Edipo sabe que está hablando de él mismo y no solo que él mismo es el hablante. Por tanto, la autoconciencia propia de un uso de "yo" queda inexplicada por la autoconciencia del hablante consistente en el conocimiento que tiene el hablante de él mismo como realizador de la emisión. Así que, después de todo, el concepto de autoconciencia como el conocimiento que tiene el hablante de que él mismo realiza la emisión no es suficiente para que haya la autoconciencia propia de un uso de "yo". En realidad, no estoy siendo totalmente justo con la posición de O’Brien, principalmente en su versión más reciente y completa (cf. O’Brien 2007, pp. 74-88). O’Brien, afinando ideas de C. Peacocke (cf. 1983, pp. 133-139; 2008, pp. 78-92), propone un modelo de la autorreferencia autoconsciente consistente en que un usuario X de "yo" tiene las siguientes piezas de conocimiento:

a) X sabe que él mismo es el usuario de "yo" (condición pragmática)

b) X sabe que el referente de "yo" en cada contexto es el usuario de "yo" en el contexto (condición semántica).

Así que en efecto,

c) X sabe que su uso de "yo" refiere a él mismo

Parece que entonces Peacocke y O'Brien logran explicar que el hablante tenga el conocimiento de estar hablando de él mismo. Pero hay un problema previo sin resolver: dar una explicación de la autoconciencia consistente en (a) el conocimiento que tiene el hablante, bajo un modo de presentación de primera persona, de que él mismo es el usuario de "yo". El resultado es que, como consecuencia de ello, permanece también el problema de explicar en qué consiste el modo de presentación de primera persona bajo el cual (c) el hablante sabe que está hablando de (refiriéndose a) él mismo ${ }^{5}$.

4 Si hay referencia garantizada a él, Edipo, aquí el uso de la descripción "el asesino de Layo" por parte de Edipo tiene que interpretarse como un uso atributivo, no referencial.

O’Brien trata de explicar ese modo de presentación de primera persona en términos de la conciencia de agente (agent's awareness) que es propia del hablante como realizador de 
Estas reflexiones nos conducen a la razón por la que Anscombe desestima la posibilidad de que el significado del pronombre personal "yo" venga dado por una regla de autorreferencia, incluso cuando el sentido de 'hablar de uno' es el del reflexivo indirecto. Suele aducirse que la razón esencial es que esa regla de autorreferencia introduce circularidad: en efecto, el reflexivo indirecto "él mismo" es un recurso para reproducir vicariamente el uso de "yo" en contextos intensionales, de manera que solo es comprensible en términos del significado del pronombre personal, y así la única explicación del contenido-que de la oración (oratio obliqua) "X dice que él mismo es $\mathrm{F}$ " es en términos de una oración (oratio recta) dicha por X que contenga una expresión de primera persona, "Yo soy F" o "F es una propiedad mía" o "F es verdadero de mí", etc... Pero la razón principal es una vez más que esa regla de autorreferencia introduce vacuidad. De entrada, la circularidad como tal no es tan dañina (cf. Garrett 1994, pp. 100-102). Afirmar "X piensa que él mismo es F" es adscribir a X un modo de pensar en primera persona acerca de él mismo, esto es, es atribuirle autoconciencia, lo cual es evidentemente circular en la medida en que ahora la regla de autorreferencia es realmente: "yo" es la palabra que cada persona usa para hablar de ella misma bajo un modo de presentación de primera persona; donde una explicación del uso de "yo" nos remite a un modo de pensar en primera persona. Pero esta objeción no es relevante más que bajo el supuesto de que una explicación de "yo" tiene que ser reduccionista, en el sentido de que el significado de "yo" tenga que ser analizable en términos que no presupongan un modo de pensar en primera persona. Ahora bien, no hay por qué aceptar que un análisis genuino esté sometido a esa restricción si la regla de autorreferencia no es formulada sin especificación alguna, como acaba de hacerse, sino que es formulada en términos de una especificación robusta del modo de pensar en primera-persona: "yo" es la palabra que cada persona usa para hablar de ella misma bajo tal-y-tal modo de presentación de primera persona. De esta forma, la circularidad será inerme. Pero Anscombe argumenta contra la posibilidad de evitar la vacuidad, lo cual ella expresa fregeanamente en términos de que no hay un camino de vuelta desde la referencia al sentido: cuando nosotros afirmamos "X piensa que él mismo es F", logramos determinar cuál es el referente del pensamiento de $\mathrm{X}$ al determinar quién es $\mathrm{X}$ bajo el modo de presentación de tercera persona mediante el que nosotros pensamos acerca de $\mathrm{X}$, no determinamos cuál es el referente del pensamiento de $\mathrm{X}$ bajo el modo de presentación de primera persona mediante el que X piensa acerca de él mismo. En realidad, esto es una consecuencia de lo que sucede con el uso de "yo": cuando X afirma "Yo soy F", logramos determinar cuál es el referente de "yo" al determinar quién es X bajo el

una emisión con “yo" (O’Brien 2007, pp. 114-124; 182-190). Independientemente de la evaluación que se haga de ello, resulta que la transferencia del modo de presentación de (a) a (c) entrañaría que el modo de presentación del referente de "yo" es el modo de presentación del hablante como realizador de cualquier emisión, y no solo de una emisión con "yo". Pues, como señalé anteriormente, la conciencia de agente que es propia del hablante es un factor común a la realización de cualquier emisión. De manera que no habría un modo de presentación de primera persona que fuera idiosincrásico de la realización de una emisión con "yo" y que, por tanto, pudiese explicar la autoconciencia propia de un uso de "yo". 
modo de presentación de tercera persona mediante el que nosotros pensamos acerca de X, a saber: como el emisor de la oración, no determinamos cuál es el referente de "yo" bajo el modo de presentación de primera persona mediante el que X, solamente cuando usa "yo", piensa acerca de él mismo. La tesis fregeana de Anscombe es que no tenemos acceso al modo de pensar en primera persona de X.

Así que alcanzamos el siguiente resultado. Parecía que la regla de autorreferencia, en el sentido de 'hablar de uno' con el reflexivo indirecto, podía dar el significado de "yo" al explicar la autoconciencia del hablante en términos de un modo de presentación de primera persona. Pero ello demandaba una explicación no vacua de en qué consiste el conocimiento que tiene el hablante de él mismo bajo ese modo de presentación. Ahora podemos decir que la razón por la que la regla de autorreferencia no da el significado de "yo" es que propiamente no puede especificar, en términos de un modo de presentación de primera persona, el conocimiento que tiene el hablante de estar hablando de él mismo: no puede especificar en qué consiste hablar de uno mismo bajo tal-y-tal modo de presentación de primera persona.

\section{El caso del usuario de "A"}

a) De la autoconciencia al reflexivo directo

En la discusión sobre el caso del usuario de "A" es donde se pone en juego un concepto robusto de autoconciencia, pues en el contraste entre un uso de "yo" y un uso de "A" se revela la naturaleza de la autoconciencia expresada por un uso genuino de "yo".

Anscombe escribe:

Imaginemos una sociedad en la que todos están etiquetados con dos nombres. Uno figura en su espalda y en la parte superior de su pecho, y estos nombres, que sus portadores no pueden ver, son varios: de la "B" a la "Z", por decir. El otro, "A", está estampado en el interior de sus muñecas, y es el mismo para todos. Al hacer informes sobre las acciones de los demás todos usan el nombre sobre su pecho o su espalda si pueden ver estos nombres o están acostumbrados a verlos. Además, todos aprenden a responder a emisiones del nombre sobre su propio pecho y espalda en el modo y en el tipo de circunstancias en las que nosotros tendemos a responder a emisiones de nuestros nombres. Informes sobre las propias acciones de uno, que uno da a partir de la observación, se hacen usando el nombre sobre la muñeca (Anscombe 1981, p. 24) .

$6 \quad$ El texto sigue: "Tales informes se hacen, no sólo sobre la base de la observación, sino también sobre la base de la inferencia y el testimonio u otra información. Por ejemplo, B deriva conclusiones expresadas por oraciones con 'A' como sujeto a partir de los enunciados de los demás con 'B' como sujeto". 
De entrada, cada hablante de esta sociedad tiene dos nombres que refieren a él: uno equivalente a un nombre propio (por ejemplo, "B") y otro, "A", que solo es usado por cada hablante para hablar de él mismo. Entonces, parecería que "A" y "yo" son palabras que tienen el mismo rol, en concreto, por el hecho de que el significado de "A" es caracterizado en términos de una regla de autorreferencia. Pero, ¿de qué regla de autorreferencia se trata, en qué sentido B 'habla de él' mediante un uso de "A"? El siguiente comentario de Anscombe no deja lugar a dudas:

Puede preguntarse: ¿qué quiere decir 'informes sobre las propias acciones de uno'? Establezcamos que quiere expresar, por ejemplo, los informes sobre las acciones de B que proceden de la boca de B. Esto es, los informes desde la boca de $\mathrm{B}$ diciendo que A hizo tal-y-tal cosa son prima facie verificados al descubrir que B lo hizo y son definitivamente falseados al encontrar que no lo hizo ( Ibíd.).

Lo cierto es que esta caracterización es satisfecha tanto por un uso de "A" como por un uso de "yo". Si B dijera "Yo estoy levantando el brazo", también estaríamos ante un informe sobre una acción de B que procede de la boca de $\mathrm{B}$ y es verificado o falseado al descubrir que B lo está o no lo está haciendo. La cuestión es que en el caso de un uso de "A" se supone que ésta es una caracterización completa de lo que significa 'informes sobre las propias acciones de uno'. Pero si todo en lo que consiste un informe de B sobre su propia acción en términos de un uso de "A" es en informar sobre una acción de $\mathrm{B}$ que procede de la boca de $\mathrm{B}$, entonces el sentido en el que B 'habla de él' mediante un uso de "A" es el del reflexivo directo: si B dice "A está levantando el brazo" y ese informe solo puede ser entendido como un informe sobre una acción de $\mathrm{B}$ que procede de la boca de B, el sentido en el que B habla de él no es, con todo, un sentido tal que B conozca que él mismo es la persona de la que está hablando. Si B dice que A está levantando el brazo, con todo, la oración con el reflexivo indirecto "B dice que él mismo está levantando el brazo" es falsa. De nuevo, estamos ante un hecho semántico que debe ser explicado en términos de que un uso de "A" no cuenta como una expresión de autoconciencia, y una vez más no es una explicación genuina apelar simplemente al dato de que B no tiene el conocimiento de que está hablando de él mismo.

En principio, da la impresión de que Anscombe, contrariamente a lo que señalé, considera que la diferencia entre un uso de "A" y un uso de "yo" es debida a que "yo" está gobernado por la regla de autorreferencia en el sentido del reflexivo indirecto, en la medida en que un uso de éste involucra trivialmente la expresión de autoconciencia. Esta reflexión está basada en la sustitución de los dos sentidos de 'hablar de uno' por dos sentidos de 'tener conciencia de uno'. Anscombe escribe:

Ellos (los usuarios de "A") quizá no tienen autoconciencia, aunque cada uno conoce bastante acerca del objeto que él (de hecho) es; y tiene un nombre, el mismo que cualquier otro tiene, que usa en informes acerca del objeto que él (de hecho) es.

Precisamente por esa razón, esto -que ellos no tienen autoconciencia-parece no ser verdadero. B es consciente de, esto es, él observa, algunas actividades de B, esto es, que son suyas. Él usa el nombre "A”, como todos lo hacen, para referirse a él mismo. Por tanto, él es consciente de él mismo. Por tanto, él tiene autoconciencia. 
Pero cuando hablamos de autoconciencia no queremos decir eso. Queremos decir alguna cosa que se manifiesta en el uso de "yo" como opuesto al uso de "A".

La expresión "autoconciencia" puede ser convenientemente explicitada como 'conciencia de que tal-y-tal es verdadero de uno mismo'. (...) "uno mismo" no es aquí sino el reflexivo indirecto: esto es, el reflexivo del discurso indirecto (Anscombe 1981, pp. 24-25).

El movimiento que Anscombe estaría realizando aquí aún mostraría más la vacuidad de apelar a la expresión de autoconciencia mediante el reflexivo indirecto. Y es que ahora la vacuidad estaría infectada de un nuevo tipo de circularidad. La idea sería: la diferencia entre que el reflexivo indirecto realmente sea una expresión de autoconciencia y el reflexivo directo no lo sea está en que una locución 'tener conciencia de uno' que sirva para cualificar el sentido de 'hablar de uno' con el reflexivo indirecto incluye un uso del reflexivo indirecto y, en cambio, una locución análoga que cualifica el sentido de 'hablar de uno' con el reflexivo directo incluye un uso del reflexivo directo. Así, introduzcamos una regla de autorreferencia tratando de explicar en qué consiste que el reflexivo indirecto sea un expresión de autoconciencia, la regla: "yo" es la palabra que cada persona usa para hablar de ella misma autoconscientemente, es decir, siendo consciente de ella misma. Es evidente que es una regla circular en la medida en que trata de explicar en qué consiste que el reflexivo indirecto ('hablar de ella misma') sea una expresión de autoconciencia mediante un segundo uso del reflexivo indirecto ('consciente de ella misma'), que obviamente queda sin explicar. De esta manera, tal regla no explica la diferencia entre que un uso de "yo" sea una expresión de autoconciencia y un uso de "A" no lo sea, porque aún faltaría una explicación de en qué consiste que el segundo reflexivo indirecto sea una expresión de autoconciencia y el segundo reflexivo directo no lo sea ${ }^{7}$.

Pero es que Anscombe no seguía realmente este camino, sino que ya al presentar su sociedad imaginaria pone en juego, por contraste, un concepto robusto de autoconciencia. La diferencia entre que un uso de "yo" sea una expresión de autoconciencia y un uso de "A" no lo sea consiste en que si B informara con "yo" expresaría una concepción inmediata o no observacional de sus acciones, mientras que si B informa con " $\mathrm{A}$ " expresa una concepción observacional de las mismas: en efecto, Anscombe ha dicho que con "A", B informa sobre sus propias acciones a partir de la observación, o B tiene el punto de vista del observador, en el sentido de que su informe "A está levantando

7 Decir que la locución 'consciente de ella' con el reflexivo directo no expresa la autoconciencia porque hay un sentido en el que Juan es consciente de (conoce a) la persona Juan pero no es consciente de que él mismo es la persona Juan a quien conoce, no es explicar en qué consiste que el reflexivo directo no sea una expresión de autoconciencia sino que es volver a exponer la diferencia semántica entre el reflexivo indirecto y el directo. Con respecto a la locución con el reflexivo directo podemos decir que, en vez de autoconciencia, tenemos autoconocimiento: "Autoconocimiento es conocimiento del objeto que uno es, del animal humano que uno es" (Anscombe 1981, p. 34). Pero seguimos sin explicar qué es lo que está faltando para que haya autoconciencia. 
el brazo" está basado en la observación de que B está levantando el brazo; mientras que con "yo", B informaría sobre sus propias acciones sin ser un observador de las mismas sino, más bien, teniendo el punto de vista del agente, en el sentido de que su informe "Yo estoy levantando el brazo" estaría basado en el hecho mismo de que B está levantando el brazo.

Podemos establecer una comparación con la distinción entre el uso de "yo" como sujeto - por ejemplo, en las oraciones "Yo intento levantar mi brazo", "Yo tengo dolor de muelas"- y el uso de "yo" como objeto - por ejemplo, en las oraciones "Mi brazo está roto", "Yo he crecido cinco centímetros"-, tal y como esta distinción es introducida por Wittgenstein (cf. 1968, pp. 100-101). Hay que decir dos cosas al respecto. La tesis de Anscombe, estando en cuestión la referencialidad de "yo" en general, es que todos los usos genuinos de "yo" son una expresión de autoconciencia, es decir, de una concepción inmediata, en cuyo caso resulta que, por un lado, las concepciones inmediatas cubren tanto las autoadscripciones psicológicas mencionadas como las autoadscripciones físicas en "Yo estoy levantando el brazo", "Mi brazo está roto", "Tengo un chinchón en la frente", siempre que tales informes no estén basados en la observación; y, por otro lado, habrá que considerar que tanto las autoadscripciones psicológicas en "Yo tengo dolor de muelas, lo dice el escáner" como las autoadscripciones físicas en "Yo he crecido cinco centímetros", "Mi brazo está roto, lo veo", no expresan concepciones inmediatas y, por tanto, los usos de "yo" no son genuinos. O tal vez sea mejor decir: la clase de las concepciones inmediatas y la clase de los usos de "yo" como sujeto tienen la misma extensión bajo la restricción de que es la perspectiva de la primera persona, no observacional, y no la naturaleza psicológica (pero no física) de las autoadscripciones, lo que define un uso de "yo" como sujeto, esto es, un uso genuino de "yo" en el caso de una autoadscripción psicológica como "Yo tengo dolor de muelas", el sujeto B de la autoadscripción no tiene el punto de vista de un agente, pues no está informando de su acción. Lo relevante es que la autoadscripción no esté basada en la observación, o introspección en este caso, de que B tiene un dolor de muelas, sino en el hecho mismo de que B tiene ese dolor. Además, para Wittgenstein lo idiosincrásico de un uso de "yo" como sujeto es que expresa una concepción sin-sujeto, por así decirlo, de los propios estados y acciones: una concepción sin-sujeto es aquella que es formada sin que tenga lugar el reconocimiento o identificación de la persona particular que siente, piensa o actúa y, por tanto, sin que haya la posibilidad de equivocarse con respecto al poseedor de los estados y al agente de las acciones, quien es uno mismo. La idea de lo que es una concepción sin-sujeto puede entenderse mejor por contraste

$8 \quad$ En otras palabras, parece que según la distinción entre uso como sujeto y uso como objeto la expresión de autoconciencia dependería de la naturaleza de la acción o estado adscrito (mental versus material), es decir, de la ontología, y, en cambio, según la distinción entre expresar una concepción inmediata y expresar una concepción mediata, la expresión de autoconciencia dependería de la naturaleza de la adscripción (no observacional versus observacional), es decir, de la epistemología. 
con lo que es una concepción con-sujeto. Anscombe narra y comenta así el siguiente relato de William James:

“Estábamos transitando en un vagón; la puerta se abrió y X, alias 'Baldy', cayó sobre el camino. Paramos inmediatamente, y entonces él preguntó ‘¿Cayó alguien?' o ‘¿Quién cayó?’ -No recuerdo las palabras exactamente. Cuando se le contó que Baldy cayó, él dijo ‘¿Cayó Baldy? Pobre Baldy!’” (...).

Lo que indujo a alguien a darle información en la forma "Baldy cayó" fue, supongo, que su comportamiento ya mostraba el lapso de autoconciencia, como James lo llamó. Él acababa de caer del carruaje, era consciente y tenía la idea de que alguien había caído del carruaje - o conocía que alguien había caído pero se preguntaba quién (...).

Resultaba que su pensamiento del suceso, la caída del carruaje, era un suceso para el que él buscaba un sujeto, su captación del suceso era la de algo que requería un sujeto (...). Él no tenía lo que yo llamo 'concepciones inmediatas -del agente o paciente- de acciones, sucesos y estados'. Éstas son concepciones sin-sujeto. Es decir, no entrañan la conexión de lo que es comprendido mediante un predicado con un sujeto concebido distintamente (Anscombe 1981, p. 36).

Por tanto, el quid del relato de James es que Baldy padeció un lapso de autoconciencia consistente en que para él el suceso que le había ocurrido demandaba la identificación de esa persona particular llamada "Baldy", de hecho él mismo, con lo que su pensamiento del suceso era una concepción con-sujeto del suceso. Sin embargo, en el último párrafo citado Anscombe añade algo más a la tesis wittgeinsteiniana. Según ella, una concepción sin-sujeto de un estado o acción no es solo una concepción que no está basada en la identificación del sujeto del estado o acción, sino que es tal que no hay concepción alguna del sujeto: no hay 'la conexión de lo que es comprendido mediante un predicado con un sujeto concebido distintamente" . La conclusión es que un uso genuino de "yo" expresa la autoconciencia del hablante en el sentido de que expresa una concepción inmediata, no observacional, de su estado o acción que no incluye concepción alguna del sujeto de ese estado o acción.

Pues bien, el resultado es, a mi modo de ver, que Anscombe logra así explicar en qué consiste la expresión de autoconciencia mediante un uso de "yo" sin tener que hacerlo mediante un recurso a la diferencia semántica entre el uso del reflexivo directo y el uso del reflexivo indirecto. La expresión de autoconciencia va a depender de que haya una concepción inmediata y sin-sujeto de un estado o acción, y eso significa para Anscombe que no hay además una concepción de primera persona del sujeto del estado o acción. Es la perspectiva de primera persona, no observacional, acerca del estado o acción, y no una perspectiva de primera persona acerca del sujeto, lo que hace que un uso de "yo" sea una expresión de autoconciencia. Es posible argumentar que la expresión de autoconciencia requiere también adscribir al hablante un modo de pensar

9 Me detendré nuevamente en este punto al ocuparme, en 2c, del diagnóstico general del error de Anscombe sobre la relación entre las dimensiones semántica y epistémica de "yo". 
en primera persona acerca de él mismo, un modo de pensar que no esté basado en el reconocimiento o identificación del sujeto. Pero, de que haya un modo de presentación no observacional de un estado o acción y de que no haya un modo de presentación del sujeto que esté basado en la identificación, no se sigue que haya un modo de presentación del sujeto que no esté basado en la identificación. Más aún, como acabo de señalar, Anscombe afirma explícitamente que una concepción sin-sujeto excluye cualquier modo de presentación del sujeto. De entrada, esto permite que la cuestión semántica quede liberada, por así decirlo, para un tratamiento independiente: la cuestión de si "A" es una palabra autorreferencial como lo sería "yo" debe dilucidarse en los términos semánticos originalmente formulados, relativos exclusivamente a "hablar de uno' y no a 'tener conciencia de uno'. Como un uso de "yo" expresa una concepción sin-sujeto, por ejemplo, de la acción de levantar el brazo, no cabe decir que el sujeto de la autoadscripción es consciente de que él mismo está levantando el brazo. Pero, entonces, tampoco cabe decir que el usuario de "yo" esté hablando de él mismo en el sentido del reflexivo indirecto. De manera que la cuestión de si "A" es una palabra autorreferencial como lo sería "yo" puede dilucidarse en términos de si satisface una regla de autorreferencia con el reflexivo directo, pues no hay nada que vuelva un sinsentido la posibilidad de que la expresión de autoconciencia con el uso de "yo" sobrevenga en la fijación de la referencia de "yo" mediante la regla de autorreferencia con el reflexivo directo: "yo" es la palabra que cada persona usa para hablar de ella (reflexivo directo), el usuario de "yo", siendo consciente de forma inmediata, o no observacional, solo de su estado o acción. Por ejemplo, si B dijera "Yo estoy levantando el brazo", estaría usando "yo" para hablar de B, el usuario de "yo", siendo consciente de forma inmediata solo de la acción de levantar el brazo; no estaría usando "yo" para hablar de él mismo autoconscientemente, es decir, siendo consciente de forma inmediata de que él mismo está realizando la acción de levantar el brazo.

b) La referencia garantizada: la dimensión semántica.

Por tanto, la cuestión semántica es: ¿"A" es una palabra autorreferencial como lo podría ser "yo", en el sentido de que satisface la regla: "A" es la palabra que, en la sociedad imaginaria, cada persona usa para hablar de ella (reflexivo directo), el usuario de "A"? Antes di por supuesta la caracterización de Anscombe de que un informe de B sobre su propia acción mediante un uso de "A" es un informe sobre una acción de $B$ que procede de la boca de B. Esto es una verdad trivial en la medida en que un informe de B sobre una acción de B es obviamente un informe de B sobre su propia acción -aunque B no sepa que es suya. También, bajo el supuesto de que A y B son la misma persona cuando B está usando "A", es una verdad trivial decir que un informe de $\mathrm{B}$ sobre una acción es un informe sobre una acción de $\mathrm{B}$; de modo que si $\mathrm{B}$ dice que A está levantando el brazo, entonces la oración "B dice que él ${ }^{10}$ está levantando 
el brazo" es verdadera. Pero lo que no es trivial en absoluto es ese supuesto implícito de identidad. Quedó claro que uno aprende a usar " $A$ " para informar sobre sus propias acciones observando que " $A$ " está estampado en su muñeca al observar el cuerpo de quien actúa. De nuevo, es una verdad trivial que un informe de B sobre una acción mediante un uso de " $A$ " a resultas de observar que "A" está estampado en su muñeca -aunque B no sepa que es suya- es un informe sobre una acción de B. Pero el hecho es que, de entrada, no hay ninguna garantía de que un informe de B sobre una acción sea el resultado de observar que "A" está estampado en su muñeca y no en la de algún otro: puesto que todos los hablantes tienen estampado "A" en la muñeca, el informe de B sobre esa acción puede ser el resultado de un error de identificación de su muñeca al observar el cuerpo de alguna otra persona actuando, en cuyo caso ese informe no es un informe sobre una acción de B.

$\mathrm{Y}$ entonces es evidente que, con respecto a un error de identificación, nada se soluciona apelando al hecho de que un informe de B sobre una acción es el resultado de observar el cuerpo de quien actúa tal y como es presentado, tanto limitada como privilegiadamente a B. Anscombe escribe:

Así, para cada persona hay una persona de quien tiene perspectivas tanto característicamente limitadas como característicamente privilegiadas: a no ser en espejos ella nunca ve la persona entera, y sólo puede lograr perspectivas más bien especiales de lo que ve. Algunas son particularmente buenas y otras son particularmente malas (Anscombe 1981, p. 24).

No parece que, como dice Anscombe, haya una sola persona, o de haberla solo la hay contingentemente, de quien el hablante pueda tener perspectivas tanto limitadas como privilegiadas. Por lo que hace al carácter privilegiado, lo cierto es que no hay ninguna garantía de que un informe de B sobre una acción sea el resultado de observar desde algún punto de vista privilegiado que "A" está estampado en su muñeca y no en la de algún otro: puesto que hay circunstancias en las que una muñeca ajena puede parecer caer bajo la observación privilegiada de uno, exactamente como la propia, el informe de B sobre esa acción puede ser el resultado de un fallo de identificación de su muñeca, en cuyo caso ese informe no es un informe sobre una acción de B. Por lo que hace al carácter limitado, Anscombe escribe:

Desde luego, algunas veces un hombre B puede cometer un error al ver el nombre "A" en la muñeca de otro, y no darse cuenta de que es la muñeca de un hombre cuyo otro nombre no es finalmente inaccesible para B de esa manera especial en que su propio nombre ("B") lo es ( Ibíd.).

Así, por lo que hace al carácter limitado, no hay ninguna garantía de que un informe de B sobre una acción sea el resultado de observar que "A" está estampado en su muñeca y no en la de algún otro, al ser inobservables para $\mathrm{B}$ determinadas partes del cuerpo que lo identificarían como suyo, en particular, su pecho y su espalda, que es donde figura el otro nombre ("B"). Anscombe misma señala que, como en lo que atañe a la observación de una muñeca no hay que darse cuenta de otras partes difícilmente 
observables del cuerpo, el informe de B sobre esa acción puede ser, una vez más, el resultado de un error de identificación de su muñeca y, por tanto, ese informe puede ser un informe sobre una acción de, digamos, C.

Ahora bien, aquí lo que tenemos es un problema epistémico: la posibilidad de un error de identificación. Lo preocupante para Garrett es que esa posibilidad pondría en entredicho que B hiciera referencia a él mediante un uso de "A", es decir, que no hay garantía de que B hable de él con un uso de "A": como si, porque contingentemente $B$ puede cometer un error de identificación de su muñeca cuando usa "A", también contingentemente $\mathrm{B}$ pudiera referirse correctamente a alguna otra persona cuando usa "A". Pero, como mostraré a continuación, estamos ante una flagrante transgresión de la independencia entre las dimensiones semántica y epistémica del uso de "A", consistente en no distinguir un sentido netamente semántico de referencia garantizada y la garantía epistémica frente a un error de identificación. La tesis netamente semántica de Anscombe es que "A" es una palabra indistinguible de "yo" con respecto a la satisfacción de la siguiente regla: si X asevera algo con "yo" como sujeto de la oración, su aserción será verdadera si, y sólo si, lo que asevera es verdadero de X. Esta es otra forma de decir que "A" es una palabra indistinguible de "yo" con respecto al siguiente principio salva veritate: una ocurrencia de "yo" en una oración "Yo soy F" emitida por X puede ser reemplazada salva veritate por un nombre de X (por ejemplo, " $X$ ”); de donde se sigue un enlace entre los valores de verdad de distintas oraciones: una oración "Yo soy F" emitida por X es verdadera si, y solo si, la oración "X es F" es verdadera. El sentido en el que "A" satisface la regla es el sentido semántico en el que Anscombe habla de referencia garantizada ${ }^{11}$ como garantía de la necesaria existencia del referente: la idea es que el referente de una palabra " $X$ " debe existir porque si $X$ es el usuario de " $X$ ", entonces necesariamente $X$ existe. De manera que el referente de un uso de " $A$ " debe existir porque si B, cuando A es B, es el usuario de "A", entonces necesariamente B existe. Anscombe escribe:

De este modo, suponiendo que conozco a alguien llamado "X" y llamo a una cosa "X" con la intención de referirme a esa persona, una garantía de referencia en este sentido sería una garantía de que hay tal cosa como X. El nombre "A" que inventé tendría este tipo de referencia garantizada. El usuario de "A" quiere hablar de un

11 Anscombe (cf. 1981, pp. 29-30) introduce tres sentidos en los que podría decirse que una palabra " $\mathrm{X}$ " tiene referencia garantizada, y su idea es que si "yo" es una palabra referencial satisface los tres sentidos y, en cambio, "A" solo satisface los dos primeros, en cuyo caso un uso de "yo" y un uso de "A" serían referencialmente distinguibles. El primero es (i) el sentido en el que el usuario de " $\mathrm{X}$ " debe existir, pues de otro modo no estaría haciendo un uso de " $\mathrm{X}$ ". Esto es trivial: obviamente no hay ninguna diferencia entre " $\mathrm{A}$ " $\mathrm{y}$ "yo" porque no hay ninguna diferencia entre "A", "yo" y cualquier otra palabra, ya que el usuario de cualquier palabra debe existir pues de otro modo no estaría usándola. El segundo es (ii) el sentido en el que el referente de " $X$ " debe existir, pues de otro modo el usuario no estaría haciendo un uso de "X". Este es el que discuto en el texto. De (iii) me ocupo después, en 2c. 
cierto ser humano, uno que cae bajo su observación de un modo más bien especial. Esa persona es él mismo y, por ello, dado que él ha captado el uso de "A", él no puede menos que estar hablando de una persona real (Anscombe 1981, p. 30).

Lo que Anscombe está diciendo es, pues, que el referente de un uso de "A" debe existir porque si la persona que cae bajo la observación especial del usuario es precisamente el usuario de "A", entonces necesariamente esa persona existe. Pero la afirmación no es tan trivial como pudiera parecer, ya que si la garantía de la necesaria existencia del referente depende de que esté garantizada la identidad entre el referente y el usuario de "A", a su vez la garantía de esa identidad no está asegurada de antemano. Supongamos que el usuario de "A" tiene la intención de referirse a quienquiera que cae bajo su observación especial, esto es, supongamos que el uso de "A" es un uso atributivo de la descripción "la persona que cae bajo la observación especial del usuario de "A": en ese caso, no hay una garantía de la identidad entre el referente y el usuario porque es algo contingente que la persona que cae bajo la observación especial (en su carácter tanto privilegiado como limitado) del usuario de " $\mathrm{A}$ " sea precisamente el usuario de "A" ${ }^{12}$. Así, si la persona que cae bajo la observación especial del usuario de "A" es $\mathrm{C}$, cuando el usuario de "A" es B, no es verdad que el referente de "A" deba existir porque, como la persona $\mathrm{C}$ que cae bajo la observación especial del usuario de " $\mathrm{A}$ " es precisamente el usuario de "A" -al contrario, precisamente no lo es!-, entonces necesariamente esa persona existe. Por lo tanto, la referencia garantizada de un uso de "A", es decir, la garantía de la necesaria existencia del referente, no puede venir dada por el uso atributivo de una descripción. Supongamos ahora que el usuario de "A" tiene la intención de referirse a la persona $\mathrm{B}$, cuando el usuario de " $\mathrm{A}$ " es $\mathrm{B}$, esto es, supongamos que el uso de "A" es un uso referencial de la descripción "la persona que cae bajo la observación especial del usuario de "A". Es fácil imaginar que el usuario ha aprendido o captado el uso de "A" formando la intención de referirse a la persona, precisamente él, que en las ocasiones paradigmáticas del aprendizaje de la palabra caía bajo su observación especial, de modo que en adelante usa " $\mathrm{A}$ " con la intención de referirse a esa persona, y no a alguna otra, caiga o no caiga entonces bajo su observación especial ${ }^{13}$. En ese caso resulta que, aún siendo algo contingente que la persona que cae

12 De hecho, Anscombe basa la referencia garantizada en un uso atributivo de la descripción. La idea sería que solo hay una persona que pueda caer bajo la observación especial del usuario, el usuario mismo, aunque pueda haber un error de identificación de su muñeca. Ciertamente, en cada contexto de uso de la descripción "la persona que cae bajo la observación especial del usuario de "A", solo hay una persona que satisface la descripción. Pero si es un caso de un error de identificación, ¿no cae precisamente esa otra muñeca, y con ella la persona a la que pertenece, bajo la observación especial del usuario de "A"? De manera que en ese contexto de uso la descripción definida (en sentido atributivo) no sería satisfecha por el usuario de "A".

13 Es habitual interpretar a K. Donnellan como si introdujese la noción de uso referencial en términos de referirse a un objeto que no tiene que satisfacer la descripción pero que está conectado informacionalmente a la Evans con el hablante en el contexto de uso. Por 
bajo la observación especial del usuario de "A" sea precisamente el usuario de "A", está garantizada la identidad entre el referente y el usuario, como Anscombe quería, porque hay una referencia garantizada a la persona que es el usuario de "A": aunque la persona que de hecho cae bajo la observación especial del usuario de "A" sea $\mathrm{C}$, cuando el usuario es $\mathrm{B}$, es verdad que el referente de "A" debe existir porque, como la persona $B$ que en el pasado cayó bajo la observación especial del usuario de "A" es precisamente el usuario de "A", entonces necesariamente esa persona existe.

Tenemos que el usuario de "A" puede cometer un error de identificación de su muñeca porque es algo contingente que la persona que cae bajo su observación especial al observar así una muñeca sea él, el usuario de "A". Esta consideración es utilizada por Garrett para introducir la posibilidad de un error de referencia, en el sentido de que contingentemente el usuario de " $\mathrm{A}$ " podría referirse correctamente a alguna otra persona cuando la persona que cae bajo su observación especial al observar así una muñeca no es él. Por eso, Garrett concluye que "A" es una palabra distinguible de "yo" con respecto a la satisfacción de la regla presentada anteriormente: no se sigue que si B asevera algo con "A" como sujeto de la oración, su aserción será verdadera si, y solo si, lo que asevera es verdadero de B. Porque si B comete un error de identificación de su muñeca y la confunde con la de algún otro, digamos $\mathrm{C}$, entonces el uso de " $\mathrm{A}$ " refiere correctamente a C, y si B asevera algo con "A" como sujeto, su aserción será verdadera si, y solo si, lo que asevera es verdadero de C (cf. Garrett 1998, p. 107). Así, una oración como "A está levantando el brazo" será verdadera en esa ocasión: C es por hipótesis quien está levantando el brazo y la oración "A está levantando el brazo" es verdadera en esa ocasión si, y solo si, C está levantando el brazo. Ahora bien, esta forma de evaluar la satisfacción de la regla puede depender justamente de concebir atributivamente el uso de "A": si un uso de "A" es el uso atributivo de la descripción "la persona que cae bajo la observación especial del usuario de "A" y B, el usuario de "A", comete un error de identificación de su muñeca al confundirla con la de algún otro, $\mathrm{C}$, que es, por tanto, en esas circunstancias, la persona que cae bajo la observación especial del usuario de " $\mathrm{A}$ " - que es en esas circunstancias quien satisface la descripción-, entonces el uso de "A" refiere correctamente a C. Por el contrario, si un uso de "A" es el uso referencial de la descripción "la persona que cae bajo la observación especial del usuario

el contrario, introduzco el uso referencial de "A" en términos de referirse a un objeto, en este caso una persona (el hablante), que en otro contexto sería la persona que satisface la descripción, pero que no está conectado informacionalmente con el hablante (con él mismo!) en el contexto de uso, donde realmente lo que está conectado así es otra persona que en ese contexto satisface la descripción. De esta manera, la referencia no estaría basada en la información sensible al contexto, sino en los usos pasados, principalmente los habidos durante el aprendizaje del uso de la palabra. Ahora bien, lo cierto es que Donnellan ya contempla la posibilidad de que el uso referencial de una descripción esté basado en el recuerdo, el testimonio, etc. En esta línea, hay argumentos en el sentido de que es suficiente para un uso referencial que el objeto referido haya sido introducido en algún momento del pasado mediante esa descripción (cf. Jeshion 2001). Debo estas consideraciones a Manuel García-Carpintero. 
de "A", la posibilidad de un error de identificación no cuenta como la posibilidad de una referencia correcta a alguna otra persona que el usuario de "A": si B, el usuario de "A", comete un error de identificación de su muñeca al confundirla con la de C, que no es la persona $\mathrm{B}$ que en el pasado cayó bajo la observación especial del usuario de "A"-que no es a quien refiere el uso referencial de la descripción-, entonces el uso de "A" refiere correctamente a B y no a C. Y, en efecto, a pesar del error de identificación, si B asevera algo con " $\mathrm{A}$ " como sujeto de la oración, su aserción será verdadera si, y solo si, lo que asevera es verdadero de B. Pero una oración como "A está levantando el brazo" será falsa en esa ocasión: C es por hipótesis quien está levantando el brazo, y la oración "A está levantando el brazo" es verdadera en esa ocasión si, y solo si, B está levantando el brazo.

De lo anterior se sigue que Garrett no respeta la independencia entre las dimensiones semántica y epistémica de un uso de "A", mientras que Anscombe sí podría hacerlo argumentando que un error de identificación con respecto a la persona que es el usuario de "A" es compatible con una referencia garantizada a esa persona. Luego, hay un sentido en el que "yo" $y$ " $A$ " son palabras indistinguibles con respecto a la capacidad del hablante para referirse correctamente a él, que es el sentido en el que la regla propuesta por Anscombe, formulada ahora así, es válida:

(Regla de referencia garantizada) Si X asevera algo con "yo" o con "A" como sujeto de la oración, su aserción será verdadera si, y solo si, lo que asevera de una manera semánticamente determinada del usuario de "yo" o de la persona que en el pasado cayó bajo la observación especial del usuario de "A", respectivamente, es verdadero de $\mathrm{X}$.

Con todo, y esta es la baza a jugar por Garrett, hay un sentido en el que "yo" $y$ "A" no son palabras indistinguibles con respecto a la capacidad del hablante para autorreferirse: según la concepción indéxica, la referencia de "yo" es fijada por la regla de autorreferencia, pero si la referencia de "A" es fijada por un uso referencial de la descripción "la persona que cae bajo la observación especial del usuario de "A", entonces no es fijada por la regla según la cual " $A$ " sería la palabra que cada persona usa para hablar de ella (reflexivo directo), el usuario de "A", es decir, la regla según la cual una instancia de "A" referiría a quienquiera que la produjo. Si la referencia de una instancia de "A" es fijada por un uso referencial de la descripción, es cierto que esa instancia refiere correctamente a quienquiera que la produjo, el usuario de " $\mathrm{A}$ ", pues quien la produjo es la persona que cayó bajo la observación especial de quien la produjo durante el aprendizaje de la palabra. Pero la referencia de esa instancia no es fijada en términos de referirse de manera semánticamente determinada a quienquiera que la produjo: la referencia de esa instancia es fijada en términos de referirse de manera semánticamente determinada a la persona que en el pasado cayó bajo la observación especial de quien la produjo. Por el contrario, Anscombe estima equivocadamente que habiendo garantizado en términos netamente semánticos que el referente de " $A$ " es el usuario de "A" - además, según ella, mediante un uso atributivo de la descripción-, ha garantizado que la referencia de " $A$ " es fijada por la regla de autorreferencia. Pero esto no es así: de la regla anterior no se sigue una regla equivalente de carácter autorreferencial: 
(Regla de autorreferencia) Si X asevera algo con "yo" o con "A" como sujeto de la oración, su aserción será verdadera si, y solo si, lo que asevera de una manera semánticamente determinada del usuario de "yo" o del usuario de "A", respectivamente, es verdadero de $\mathrm{X}$.

Como esta regla no es válida porque con un uso de " $\mathrm{A}$ " no se habla de una manera semánticamente determinada del usuario de "A", el principio de que la expresión de autoconciencia mediante un uso de "yo" debería sobrevenir en la fijación de la referencia de "yo" mediante la regla de autorreferencia, no presenta un caso contra la concepción indéxica. Si un uso de "A", que no expresa la autoconciencia del hablante, tiene una referencia que no es fijada por la regla de autorreferencia, entonces un uso de "yo", que es una expresión de autoconciencia, la expresión de una concepción inmediata y sinsujeto de un estado o acción, puede tener una referencia que sea fijada por la regla de autorreferencia: pues entre un uso de "A" y un uso de "yo" no habría una diferencia con respecto a la expresión de autoconciencia sin que hubiera una diferencia con respecto a la fijación de la referencia ${ }^{14}$.

c) La referencia garantizada: la dimensión epistémica.

Consideremos la vía que, a partir de aquí, emprende Anscombe. Ella argumenta que, a pesar de que hasta este punto "A" y "yo" podrían ser palabras referencialmente indistinguibles, sin embargo, no son palabras referencialmente indistinguibles porque no lo son en un sentido epistémico de referencia garantizada como garantía de la identificación del referente: la idea de la garantía cuestión es que lo que el usuario X de una cierta palabra " $X$ " identifica como $X$ necesariamente es $X$, el referente de la palabra $^{15}$. Es evidente, como he estado señalando, que un uso referencial de "A" no tiene esta referencia garantizada: no es verdad que cualquier persona que el usuario $\mathrm{X}$ de "A" identifica como A necesariamente es X, el referente de la palabra. Es posible que $\mathrm{X}$ se identifique erróneamente a sí mismo como la persona que ahora cae bajo su observación especial. Antes de evaluar la tesis de Anscombe, está la cuestión de si "yo" es realmente inmune a un error de identificación. La noción de esa inmunidad al error fue introducida por S. Shoemaker (1968/1994) para dar cuenta de los usos de "yo" como sujeto por oposición a los usos como objeto. Afirmar que un juicio "a es F" es inmune a un error de identificación es afirmar que no es posible la siguiente

14 Remito a la introducción y, especialmente, a la nota 3 sobre el concepto de sobreveniencia.

15 En correspondencia con la regla introducida para la referencia garantizada en sentido semántico (y el correspondiente principio salva veritate) ahora tenemos la regla: si $\mathrm{X}$ asevera algo con "yo" como sujeto de la oración, su aserción será verdadera si, y solo si, lo que asevera es verdadero de quienquiera que $\mathrm{X}$ identifique como él mismo. A continuación consideraré la validez de la regla. Lo que sí es evidente, por tanto, es que no es válida la regla: si X asevera algo con "A" como sujeto de la oración, su aserción será verdadera si, $\mathrm{y}$ solo si, lo que asevera es verdadero de quienquiera que $\mathrm{X}$ identifique como $\mathrm{A}$. 
situación: que el sujeto sepa que algún objeto particular es F pero cometa el error de aseverar "a es F" solo porque cree falsamente que el objeto que él sabe que es F es aquello a lo que "a" refiere. En el caso de la autoadscripción psicológica "Yo tengo una idea brillante", habría una inmunidad a un error de identificación: no es posible que al aseverar "Yo tengo una idea brillante" un sujeto sepa que alguien tiene una idea brillante pero su aserción sea con todo errónea solo porque cree falsamente que esa persona que él sabe que tiene una idea brillante es el sujeto al que "yo" refiere, es decir, el usuario de "yo". Consideremos, por contraste, un uso de "yo" como objeto donde no habría tal inmunidad al error. Supongamos que de repente me encuentro espacialmente desorientado y observo en un espejo, entre varias personas que me pasan desapercibidas, una de las cuales soy yo, a alguien, que de hecho no soy yo, levantando un brazo, y que, como resultado de mi parecido con él, asevero erróneamente "Yo estoy levantando el brazo". Este es un caso en el que sé observacionalmente que alguien está levantando un brazo pero cometo el error de aseverar "Yo estoy levantando el brazo" solo porque creo falsamente que esa persona que sé observacionalmente que está levantando el brazo soy yo, el sujeto al que "yo" refiere ${ }^{16}$. Parecería que, después de todo, hay usos de "yo" que no son inmunes a un error de identificación.

Sin embargo, como ya dije, la tesis de Anscombe al discutir la referencialidad de "yo" en general es que cualquier uso genuino de "yo" responde al hecho de que "yo" es la palabra que cada hablante usa para expresar su autoconciencia, sus concepciones inmediatas y sin-sujeto. Pero es claro que si un uso de "yo" expresa una concepción sin-sujeto es inmune a un error de identificación: como no hay concepción alguna del sujeto del estado o acción, no hay tampoco el conocimiento de que, por ejemplo, alguien está levantando el brazo y, por tanto, uno no puede creer falsamente que esa persona es el usuario de "yo" ". Supongamos ahora que al aseverar "Yo estoy levantando el brazo" un sujeto expresa una concepción inmediata que, no obstante, es una concepción con-sujeto, una creencia no observacional de que él mismo está levantando su brazo. También en este caso la autoadscripción física es inmune a un error de identificación: no es posible que al aseverar "Yo estoy levantando el brazo" el sujeto crea falsamente que la persona que él sabe no observacionalmente que está levantando el brazo es el sujeto al que "yo" refiere, es decir, él mismo. En efecto, ¡no puedo equivocarme al

16 Esto es precisamente lo que sucede con un uso referencial de "A": es posible que al aseverar "A está levantando el brazo", el usuario B de "A" sepa observacionalmente que alguien está levantando el brazo y su aserción sea con todo errónea solo porque cree falsamente que esa persona, de hecho $\mathrm{C}$, que está levantando el brazo es la persona a la que "A" refiere, es decir, la persona B que en el pasado cayó bajo la observación del usuario de "A".

17 Recientemente, F. Recanati ha propuesto la siguiente distinción entre pensamientos de primera persona (De Se): implícitos y explícitos. En el primer caso no es necesario que haya un modo de presentación del sujeto del pensamiento porque el sujeto no es un constituyente de la proposición pensada y, por tanto, el pensamiento no es acerca del sujeto. Como consecuencia de ello, esos pensamientos de primera persona son inmunes a un error de identificación (cf. 2007, pp. 145-148; 176-177; 191-194; 260-266). Esta noción de pensamiento implícito De Se se solapa con la noción de concepción sin-sujeto propuesta por Anscombe. 
identificar el sujeto que está levantando el brazo atribuyéndome en primera persona erróneamente la acción, que conozco no observacionalmente, de algún otro! Anscombe pone el ejemplo del obispo que comete un error de identificación de su rodilla al confundirla con la de una señora, pero que, no obstante, no puede cometer un error de identificación consistente en tomar la señora por él mismo (Anscombe 1981, p. 30). El diagnóstico de Anscombe no atiende a sus propias ideas en esta ocasión. Por un lado, si el obispo asevera "Mi rodilla tiembla" a partir de la observación de que tiembla la rodilla de alguien, entonces el obispo puede confundir su rodilla con la de la señora y, por ello, tomar la señora por él mismo, pero si la aserción del obispo es realizada a partir de la concepción no observacional de que tiembla la rodilla de alguien, entonces el obispo no puede confundir su rodilla con la de la señora ni, en consecuencia, cometer un error de identificación. La conclusión es que, efectivamente, los usos de "yo" que expresan la autoconciencia del hablante son inmunes a un error de identificación. Como tales usos son todos los usos genuinos de "yo" -no sintáctica sino lógicamente hablando, por así decirlo-, los usos de "yo" propiamente como objeto no son más que usos de, digamos, un demostrativo oculto: por ejemplo, un uso de "yo" en "Yo estoy levantando el brazo, lo veo" debería ser traducido en términos de "Ésa (persona) está levantando el brazo, lo veo" 18 .

La tesis epistémica de Anscombe sobre una garantía de la identificación del referente es haber logrado, mediante esa referencia garantizada, un argumento contra la referencialidad de "yo": si "yo" es inmune a un error de identificación y "A" no lo es, entonces, como "A" es una palabra autorreferencial en el sentido semántico, hay que concluir que "yo" no es una palabra autorreferencial en ese sentido. De otro modo, habría una diferencia con respecto a la identificación del referente sin que hubiera una diferencia con respecto a la fijación de la referencia. Es el mismo tipo de recurso a lo epistémico que encontramos en el principio de sobreveniencia original: si un uso de "yo" es una expresión de autoconciencia y un uso de "A" no lo es, entonces, como la referencia de "A" es fijada por la regla de autorreferencia, la referencia de "yo" no puede ser fijada por la regla de autorreferencia. De otro modo, habría una diferencia con respecto a la expresión de autoconciencia sin que hubiera una diferencia con respecto al modo como la referencia es fijada. Pues bien, semejante recurso no está a disposición de Anscombe en la medida en que, como argumenté en $2 b$, no ha probado mediante su caso del usuario de "A" que pudiera haber una palabra "A", distinta de "yo", cuya referencia esté semánticamente determinada por la regla de autorreferencia.

18 Parece que la transformación de indéxico en demostrativo en tales usos depende de que haya una oposición sujeto-objeto, de que haya una distancia que salvar, por así decirlo, para que el sujeto esté presente. Puede afirmarse que, por eso, un pensamiento demostrativo, pero no un pensamiento indéxico, demanda 'seguir la pista' -to keep the track of- del objeto referido, es decir, una capacidad de reidentificación del objeto: en cambio, con un uso de "yo" no hay una demanda de seguirse la pista a uno mismo -self-tracking- (cf. Cassam 1997, pp. 145-146; Evans 1982, pp. 205-267). 
Termino con un diagnóstico general del error de Anscombe sobre la relación entre las dimensiones semántica y epistémica de un uso de "yo". Como he señalado, ella sostiene que un uso genuino de "yo" expresa una concepción sin-sujeto de un estado o acción, no solo en el sentido wittgeinsteiniano de que no hay una cuestión sobre la identificación del sujeto de ese estado o acción, sino en el sentido más fuerte de que no hay concepción alguna del sujeto. Pero si no hay algún modo de presentación del sujeto, algún modo de pensar en él, es razonable concluir fregeanamente que el uso de "yo" no tiene referencia. De ahí que Anscombe piense que la clase de los usos de "yo" que expresan concepciones sin-sujeto y la clase de los usos que carecen de referencia tienen la misma extensión. Una lectura posible de Anscombe es hacerla decir que el hecho de que un uso de "yo" como una expresión de autoconciencia no entrañe la concepción del sujeto como un objeto identificable es lo que hace que el uso de "yo" no sea referencial. Tanto Garrett como J. McDowell (cf. Garrett 1994, pp. 112-113; 1998, pp. 107-109; McDowell 1998, pp. 364-365) estarían en lo correcto al señalar que esa tesis es una transgresión de la independencia de lo semántico porque introduce la cuestión del reconocimiento o identificación del sujeto como una condición necesaria para la referencialidad de "yo". Sin embargo, no parece que ella dé directamente ese paso. Más bien, podemos hacerla decir que el hecho de que un uso de "yo" como una expresión de autoconciencia no entrañe concepción alguna del sujeto, sea o no sea como un objeto identificable, es lo que hace que el uso de "yo" no sea referencial. Esta tesis es, como afirmé anteriormente, fregeanamente razonable. El problema real está en pasar de la tesis wittgeinsteiniana de que un uso de "yo" como sujeto excluye la identificación del sujeto, a la tesis de que el uso de "yo" como una expresión de autoconciencia no es tampoco la expresión de alguna concepción no identificadora del sujeto. Esta es la razón por la que Anscombe no distingue entre que la referencia garantizada de "A" sea fijada por un uso de la descripción identificadora "la persona que cae bajo la observación especial del usuario de " $A$ "” y que la referencia garantizada de "A" sea fijada de manera no identificadora por la regla de autorreferencia, como señalé al final de 2 b. Pues, para ella no es relevante distinguir entre una comprensión de la regla de autorreferencia según la cual la referencia garantizada de "yo" es fijada por un uso de la descripción identificadora "el usuario de "yo" y una comprensión de la regla de autorreferencia según la cual la referencia garantizada de "yo" es fijada de manera no identificadora - por ejemplo, causalmente- por esa regla.

Textualmente (Anscombe 1981, p. 36) Anscombe, comentando el relato de James sobre Baldy, establece una ecuación entre que para Baldy el suceso de la caída del carruaje "era un suceso para el que él buscaba un sujeto" y que para él la "captación del suceso era la de algo que requería un sujeto". Pero si la primera locución introduce una concepción del sujeto como un objeto empíricamente identificable (y reidentificable), la segunda locución puede interpretarse como introduciendo meramente una concepción del sujeto como un referente, sea o no sea empíricamente identificable, del que pueden hacerse determinadas predicaciones. Este es el paso problemático del que ella no da ninguna explicación. No es extraño que, a continuación, comente su afirmación de que "esas concepciones (inmediatas) son sin-sujeto" en términos de que tales concepciones "no entrañan la conexión de lo que es comprendido mediante un predicado con un sujeto 
concebido distintamente", pues 'un sujeto concebido distintamente' no tiene que ser más que un sujeto concebido como un referente, entre otros referentes concebibles, del que puedan hacerse determinadas predicaciones: no como un objeto identificable entre otros objetos identificables. Así que no es extraño tampoco que Anscombe termine su artículo diciendo que "la profundamente enraizada ilusión gramatical de un sujeto es lo que genera todos los errores que hemos estado considerando", en vez de decir simplemente que es la ilusión epistémica de un sujeto la que genera los errores en cuestión ${ }^{19}$.

\section{Referencias bibliográficas}

Anscombe, Gertrud E. M. (1981), "The First Person”, en G. E. M. Anscombe, Metaphysics and the Philosophy of Mind. Oxford: Basil Blackwell, pp. 21-36. Cassam, Quassim (1997), Self and World. Oxford: Oxford University Press. Evans, Gareth (1982), The Varieties of Reference. Oxford: Clarendon Press. Garrett, Brian (1994), “Anscombe and the First Person”, Crítica (26/78): 97-113. (1998), Personal Identity and Self-Consciousness. Londres: Routledge.

Jeshion, Robin (2001), "Donnellan on Neptune", Philosophy and Phenomenological Research (63/1): 111-135.

McDowell, John (1998), "Reductionism and the First Person", en J. McDowell, Mind, Value, and Reality. Cambridge Mass.: Harvard University Press, pp. 359-382.

O’Brien, Lucy (1994), “Anscombe and the Self-Reference Rule”, Analysis (54/4): 277-281.

(2007), Self-Knowing Agents. Oxford: Oxford University Press.

Peacocke, Christopher (1983), Sense and Content. Oxford: Oxford University Press. (2008), Truly Understood, Oxford: Oxford University Press.

Recanati, Francois (2007), Perspectival Thought. Oxford: Oxford University Press.

Shoemaker, Sidney (1968/1994), "Self-Reference and Self-Awareness", en Q. Cassam, ed., Self-Knowledge. Oxford: Oxford University Press, pp. 80-93.

Wittgenstein, Ludwig (1968), Los cuadernos azul y marrón. Madrid: Tecnos.

19 Esto no significa que con respecto a la cuestión ontológica del sujeto Anscombe esté obligada a pensar en términos de una teoría del no poseedor de las acciones o estados, como si la identidad de un estado o acción no requiriera la existencia de un sujeto. Al contrario, todo lo que dice presupone que si Juan asevera "Yo estoy levantando el brazo", la descripción neta de la acción, desde un punto de vista externo, tiene el contenido Juan está levantando el brazo, donde el sujeto de la acción es el ser humano vivo. 